\title{
A CONSTRUCTION OF A DOWKER SPACE
}

\author{
STEPHEN WATSON
}

(Communicated by Dennis Burke)

\begin{abstract}
We devise a method of building Dowker spaces from copies of Bing's example $G$. We show that, if there is a compact cardinal, then there is a $\sigma$-discrete hereditarily normal Dowker space.
\end{abstract}

In this paper, we describe a new method of constructing Dowker spaces. We use this method to show that, if there is a strongly compact cardinal, then there is a $\sigma$-discrete hereditarily normal Dowker space.

The topological definitions of this paper are standard (see [2]). A Dowker space is a normal Hausdorff topological space which is not countably paracompact. Dowker spaces with assorted properties have been constructed under various set-theoretic assumptions and even in ZFC. The reader is referred to [6] or Rudin's survey article [5]. A topological space is $\sigma$-discrete if it is the union of countably many subspaces, each of which is discrete in the subspace topology. The set-theoretic definitions of this paper are standard (see [4]). We use the Erdös notations $[\lambda]^{\mu}=\{A \subset \lambda:|A|=\mu\}$ and $[\lambda]^{<\mu}=\{A \subset \lambda:|A|<\mu\}$ and we also use Kunen's notation $F n(\lambda, \mu)=\left\{f:\left(\exists A \in[\lambda]^{<\omega}\right): f \in{ }^{A} \mu\right\}$. We say that $\kappa$ is strongly compact if each $\kappa$-complete filter is a subset of a $\kappa$-complete ultrafilter (see [3]). Subsets of $A \times B$ may be treated as relations.

The idea of the construction is quite simple. We iterate a hypothetical variation of Bing's space [1] along $\omega$ to get a Dowker space. This variation has two levels. The upper level is a discrete family of points and the lower level is a set of isolated points. These levels have equal cardinality $\kappa$ and we shall assume that there is a certain kind of countably complete filter on $\kappa$. The crucial property of the filter is that an open set which contains "most" of the upper level must contain "most" of the lower level. The filter is needed to make this notion precise. Lemma 1 describes this interaction. A surprising aspect is that the proof of normality is nontrivial.

Lemma 1. Let $X$ be a set of cardinality $\kappa$. Let $F$ be a countably complete filter on $X$. If there is a normal Hausdorff topology $\tau$ on $X \times 2$ such that $X \times\{0\}$ is a set of isolated points, $X \times\{1\}$ is a discrete family of points and such that,

Received by the editors March 1, 1989 and, in revised form, October 11, 1989.

1980 Mathematics Subject Classification (1985 Revision). Primary 54D15, 54D18, 54G20; Secondary 03E35, 03E55, 03E05. 
whenever $U$ is an open subset of $X \times 2, U^{-1}(1) \in F \Rightarrow U^{-1}(0) \in F$, then there is a $\sigma$-discrete hereditarily normal Dowker space of cardinality $\kappa$.

Proof. The iteration has $X \times \omega$ as an underlying set. The topology on $X \times \omega$ is $\tau_{\omega}=\left\{U \subset X \times \omega:(\forall n \in \omega)\left(U^{-1}(n) \times\{0\}\right) \cup\left(U^{-1}(n+1) \times\{1\}\right) \in \tau\right\}$. We can think of $X \times \omega$ as an ascending sequence of levels. Each two adjacent levels are homeomorphic, as a subspace, to $X \times 2$. A subset of $X \times \omega$ is open if and only if its intersection with each two adjacent levels is open in $X \times 2$.

$\tau_{\omega}$ is a topology on $X \times \omega$ because $\tau$ is a topology on $X \times 2$. Each $X \times \omega-\{(x, n)\}$ is an open set in $X \times \omega$ and so points are closed in $X \times \omega$.

We show that $X \times \omega$ is hereditarily normal. For each $A \subset X$, there is $O(A)$ clopen in $X \times 2$ such that $O(A)^{-1}(1)=A$. That is the content of the normality of $X \times 2$. This process can be finitely iterated. For each $A \subset X$ and $n \in \omega$, there is $O(A, n)$ clopen in $X \times(n+1)$ with the subspace topology such that $O(A, n)^{-1}(n)=A$. To see this, define $\left\{O_{i}(A, n): i \leq n\right\}$ by induction downwards on $i \leq n$ by $O_{n}(A, n)=A$ and $O_{i}(A, n)=\left(O\left(O_{i+1}(A, n)\right)\right)^{-1}(0)$ and let $O(A, n)=\bigcup\left\{O_{i}(A, n) \times\{i\}: i \leq n\right\}$. Let $H$ and $K$ be disjoint subsets of $X \times \omega$ such that $H \cap \bar{K}=\bar{H} \cap K=\varnothing$. We must separate $H$ and $K$ by open sets to demonstrate hereditary normality.

A basic fact is needed:

$$
A \subset X \wedge B \subset X \times(\omega-n) \wedge \overline{A \times\{n\}} \cap B=\varnothing \Rightarrow \overline{O(A, n)} \cap B=\varnothing .
$$

This shows that passing from $A$ to $O(A, n)$ does not add limit points at level $n$ or higher. Let us prove fact 1 . Let $K=\overline{A \times\{n\}} \cup O(A, n) . K$ is closed in $X \times \omega$ since $O(A, n)$ is clopen in $X \times(n+1)$ and each intersection of $K$ with two adjacent levels is closed. Thus $\overline{O(A, n)} \subset \overline{A \times\{n\}} \cup O(A, n)$ while $\overline{O(A, n)} \cap(X \times(\omega-n)) \subset \overline{A \times\{n\}}$.

A standard subtraction technique (see, e.g. Lemma 1.5.14 of [2]) shows that to separate $H$ and $K$ it suffices to find countably many open sets each of whose closures intersect at most one of $H$ and $K$ but which cover $H$ and $K$. Therefore, it suffices, without loss of generality, to find, for each $n \in \omega$, an open set $U$ such that $U \supset H^{-1}(n) \times\{n\}$ and $\bar{U} \cap K=\varnothing$. Now $O\left(H^{-1}(n), n\right)$ is an open set in $X \times \omega$ whose closure is disjoint from all of $K$ but the first $n$ levels (since $H^{-1}(n) \times\{n\} \subset H$ and $\bar{H} \cap K=\varnothing$, we can use fact 1 letting $A=H^{-1}(n)$ and $B=K \cap(X \times(\omega-n))$. It suffices, therefore, to find an open set which contains $H^{-1}(n) \times\{n\}$ and whose closure misses the $i$ th level of $K$ (where $i<n)$. In fact $V=O\left(H^{-1}(n), n\right)-\overline{O\left(K^{-1}(i), i\right)}$ does the trick. Applying fact 1, letting $A=K^{-1}(i)$ and $B=H^{-1}(n) \times\{n\}$, shows that $V$ contains $H^{-1}(n) \times\{n\}$ and completes the proof.

We show that $X \times \omega$ is not countably paracompact. For each $n \in \omega$, $X \times(\omega-n)$ is closed and $\bigcap\{X \times(\omega-n): n \in \omega\}=\varnothing$. Suppose that there is an open family $\left\{U_{n}: n \in \omega\right\}$ such that $U_{n} \supset X \times(\omega-n)$ for each $n \in \omega$ and 
$\bigcap\left\{U_{n}: n \in \omega\right\}=\varnothing$. We need another basic fact:

$$
A \subset X \times \omega \text { is open } \wedge i \in \omega \wedge A^{-1}(i+1) \in F \Rightarrow A^{-1}(i) \in F .
$$

To see this, apply the hypothesis on $X \times 2$ in the statement of the lemma, letting $U=\left(A^{-1}(i+1) \times\{1\}\right) \cup\left(A^{-1}(i) \times\{0\}\right)$. For each $n \in \omega, U_{n}^{-1}(n) \supset X \times\{n\} \in F$ and so, by induction downwards, using fact 2 , for each $i \leq n, U_{n}^{-1}(i) \in F$. In particular, for each $n \in \omega, U_{n}^{-1}(0) \in F$. Since $F$ is countably complete $\bigcap\left\{U_{n}^{-1}(0): n \in \omega\right\}$ is nonempty and so $\bigcap\left\{U_{n}: n \in \omega\right\}$ is nonempty.

In the next lemma, we construct the variation of Bing's space which is needed and isolate the combinatorial properties which the filter must have. In this way, we leave general topology and enter combinatorial set theory.

A review of Bing's space is useful. Throughout this paper we will ignore the distinction between the power set of a set $X$ and the set $2^{X}$ of 2-valued functions on $X$. A version of Bing's space with a discrete family of points $X$ is defined by letting the set of isolated points be $2^{2^{X}}$ and letting a neighborhood of $x \in X$ be defined for each $p \in\left[2^{X}\right]^{<\omega}$ by

$$
U_{p}(x)=\{x\} \cup\left\{y \in 2^{2^{x}}:(\forall s \in p)(y(s)=1 \Leftrightarrow x \in s)\right\} .
$$

If $A \subset X$, then an open set containing $A$ can therefore be defined by a function $\phi: A \Rightarrow\left[2^{X}\right]^{<\omega}$ by

$$
A \cup\left\{y \in 2^{2^{x}}:(\exists x \in A):(\forall s \in \phi(x))(y(s)=1 \Leftrightarrow x \in s)\right\} .
$$

This is how condition 3 of Lemma 2 is the filter condition of Lemma 1.

The most confusing aspect of the iteration will be the identification of an isolated point, a function from $2^{X}$ into 2 with a nonisolated point, an element of $X$. This identification is made in two steps. An element of $X$ is identified with a function from the cardinal $\left|2^{X}\right|$ into 2 in Lemma 2 but $\left|2^{X}\right|$ will not be identified with $2^{X}$ until much later. This means that an unspecified bijection $\pi$ between $2^{X}$ and $\left|2^{X}\right|$ must be used. $X$ is a set of functions from $\left|2^{X}\right|$ into 2. $\pi$ permits this noncircular method of defining $X$ to be a set of functions from $2^{X}$ into 2 .

The version of Bing's space defined above is not Hausdorff; there are too many isolated points. This is no problem, however, as we do not need all of the isolated points anyway. Condition 4 of Lemma 2 makes $X \times 2$ Hausdorff. It is really just a technical condition which ensures that the filter is nontrivial.

Lemma 2. Let $\kappa$ be a cardinal and let $\lambda=2^{\kappa}$. If there is $X \in\left[{ }^{\lambda} 2\right]^{\kappa}$ and $\pi: 2^{X} \rightarrow \lambda$ such that

(3) ( $\exists$ countably complete filter $F$ on $X):(\forall A \in F)\left(\forall \phi: A \rightarrow\left[2^{X}\right]^{<\omega}\right)$

$$
\{y \in X:(\exists x \in A)(\forall s \in \phi(x)) y(\pi(s))=1 \Leftrightarrow x \in s\} \in F,
$$

$$
\left(\forall x_{0}, x_{1} \in X\right)\left(\exists A \in 2^{X}\right) x_{0}(\pi(A))=1 \Leftrightarrow x_{1} \notin A,
$$


then there is a normal Hausdorff topology on $X \times 2$ such that $X \times\{0\}$ is a set of isolated points, $X \times\{1\}$ is a discrete family of points and $U$ open and $U^{-1}(1) \in F$ implies $U^{-1}(0) \in F$.

Proof. Let $U_{p}(x)=\{(x, 1)\} \cup\{(y, 0):(\forall s \in p) y(\pi(s))=1 \Leftrightarrow x \in s\}$. A base for the topology on $X \times 2$ is $\{\{(x, 0)\}: x \in X\} \cup\left\{U_{p}(x): x \in X, p \in\left[2^{X}\right]^{<\omega}\right\}$. This is a base because $p_{0}, p_{1} \in\left[2^{X}\right]^{<\omega}$ and $p_{1} \supset p_{0}$ implies $U_{p_{1}}(x) \subset U_{p_{0}}(x)$. Points are closed because $\bigcap\left\{U_{p}(x): p \in\left[2^{X}\right]^{<\omega}\right\}=\varnothing$. This is true because if $\left(x_{1}, 0\right) \in U_{p}(x)$ for each $p \in\left[2^{X}\right]^{<\omega}$ then, for each $s \in 2^{X}, x_{1}(\pi(s))=1 \Leftrightarrow$ $x \in s$ and this contradicts condition 4. $X \times\{0\}$ is a set of isolated points and $X \times\{1\}$ is a discrete family of points. To show normality, let $A$ and $B$ be disjoint subsets of $X$. Let $U=\bigcup\left\{U_{\{A\}}(x): x \in A\right\}$ and $V=\bigcup\left\{U_{\{A\}}(x): x \in\right.$ $B\}$. $U$ is an open set which contains $A \times\{1\}, V$ is an open set which contains $B \times\{1\}$ and $U \cap V=\varnothing\left(x_{0} \in A \wedge x_{1} \in B \wedge(y, 0) \in U_{\{A\}}\left(x_{1}\right) \cap U_{\{A\}}\left(x_{0}\right) \Rightarrow\right.$ $\left(x_{1} \in A \Leftrightarrow y(\pi(A))=1 \Leftrightarrow x_{0} \in A\right)$ which is a contradiction).

We show that $U$ open and $U^{-1}(1) \in F$ implies $U^{-1}(0) \in F$. For each $x \in U^{-1}(1)$, define $\phi(x) \in\left[2^{X}\right]^{<\omega}$ such that $U_{\phi(x)}(x) \subset U . U$ contains $\bigcup\left\{U_{\phi(x)}(x): x \in U^{-1}(1)\right\}$ and so $U^{-1}(0)$ contains

$$
\begin{aligned}
\bigcup\{\{y \in X: & \left.(\forall s \in \phi(x)) y(\pi(s))=1 \Leftrightarrow x \in S\}: x \in U^{-1}(1)\right\} \\
& =\left\{y \in X:\left(\exists x \in U^{-1}(1)\right):(\forall s \in \phi(x)) y(\pi(s))=1 \Leftrightarrow x \in s\right\} \in F
\end{aligned}
$$

\section{by Condition 3.}

The next lemma analyzes the combinatorics which have been isolated in Lemma 2 into a comprehensible form. The filter $F$ must be uniform to keep the technical condition 4 of Lemma 2 satisfied. The filter $F$ must be hypothesized to be as complete as the permissible size of the $\Delta$-systems which will arise in Lemma 3. This unfortunately adds another parameter $\mu$. The reader should keep in mind that $\mu$ is probably $\omega_{1}$ or $\kappa$. The author conjectures that the hypotheses of the lemma can be satisfied when $\mu=\omega_{1}$ but the application in this paper has $\mu=\kappa$. The lemma pushes a $\Delta$-system analysis of a neighborhood assignment through the combinatorics of Lemma 2.

Lemma 3. Let $\kappa$ and $\lambda$ be cardinals such that $\lambda=2^{\kappa}$. Let $\mu$ be a regular uncountable cardinal. Let $X \in\left[{ }^{\lambda} 2\right]^{\kappa}, \pi: 2^{X} \rightarrow \lambda$ and let $F$ be a $\mu$-complete uniform filter on $X$.

Suppose that if $Q \subset F n\left(2^{X}, 2\right)$ and either

$$
|Q|<\lambda \wedge \bigcup\left\{\bigcap f^{-1}(1)-\bigcup f^{-1}(0): f \in Q\right\} \in F
$$

or

(6) $|Q|=\mu \wedge$ the domains of the elements of $Q$ are pairwise disjoint 
then $\{y \in X:(\exists f \in Q) y \circ \pi \supset f\} \in F$. There must be a $\sigma$-discrete hereditarily normal Dowker space of cardinality $\kappa$.

Proof. We show that conditions 3 and 4 of Lemma 2 hold.

First we show condition 3. Let $A \in F$ and $\phi: A \rightarrow\left[2^{X}\right]^{<\omega} . \phi$ specifies a neighborhood assignment. We need more than $\phi(x)$; we need to know whether $x$ is an element of the various elements of $\phi(x)$. Define $\Psi: A \rightarrow F n\left(2^{X}, 2\right)$ by $(\forall s \in \phi(x)) \Psi(x)(s)=1 \Leftrightarrow x \in s . \Psi$ tells us what we want to know. We want to do a $\Delta$-system analysis of $\Psi$. An ordinary $\Delta$-system argument yields a subset of $A$ of large cardinality but we need to get "most" of $A$ to be mapped to elements of a fixed $\Delta$-system.

We will construct by induction a tree $T$ of height at most $\omega$ and cardinality less than $\mu$. A potential node in this tree is an ordered pair $(B, f)$ where $B$ is a subset of $A, f \in F n\left(2^{X}, 2\right)$ and $(\forall x \in B) \Psi(x) \supset f$. Let $N$ be the set of potential nodes.

The root of $T$ is $(A, \varnothing)$. Let us define the successors of an arbitrary node $(B, f)$. Let $G$ be a maximal subfamily of $\{\Psi(x)-f: x \in B\}$ with pairwise disjoint domains.

There are two possibilities. If $\varnothing \in G$ or $|G| \geq \mu$, then $(B, f)$ has no successors. Otherwise, we must find $I \in[N]^{<\mu}$ such that the first coordinates of the elements of $I$ partition $B$ into nonempty sets and such that the second coordinate of any element of $I$ extends $f$ by adding exactly one element to the domain. This one element must be chosen from the domain of an element of $G$. Each element of $I$ is an immediate successor of $(B, f) . T$ has now been defined. For example, if $\{\phi(x): x \in A\}$ have pairwise disjoint domains then the tree consists of a single node.

We need to define $\Gamma \in\left[\left[F n\left(2^{X}, 2\right)\right]^{\mu}\right]^{<\mu}$ to keep track of why nodes might have no successors. Whenever it is decided that $(B, f) \in T$ has no successors although $\varnothing \notin G$ at that time, we put a subset of $G$ of cardinality $\mu$ into $\Gamma$. Each element of $\Gamma$ must therefore have cardinality $\mu$ and so, since the cardinality of $T$ is less than $\mu$ (using $c f(\mu)>\omega), \Gamma$ is well defined. Let $E$ be the set of nodes of $T$ without successors. Let $Q$ be the set of second coordinates (each of which is a finite partial function) of elements of $E$. Since the cardinality of $T$ is less than $\mu,|Q|<\mu$.

We claim that $\bigcup\left\{\cap f^{-1}(1)-\bigcup f^{-1}(0): f \in Q\right\} \supset A$. This can be seen by following any element of $A$ up the tree. If a node $(B, f)$ has successors and $x \in B$, then some successor $(C, g)$ must be such that $x \in C$. Furthermore $\Psi(x) \supset f$ and $\Psi(x) \supset g$. The partial functions which are second coordinates of nodes grow as one moves up the tree so each $x \in A$ is associated with a node $(B, f)$ which has no successors but such that $x \in B$. The fact that $\Psi(x) \supset f$ implies $x \in \bigcap f^{-1}(1)-\bigcup f^{-1}(0)$.

Now $A \in F$ implies that $\{y \in X:(\exists f \in Q) y \circ \pi \supset f\} \in F$ by hypothesis 5 of Lemma 3. Furthermore, whenever $G \in \Gamma$, letting $G=Q$ in hypothesis 6 
of Lemma 3, we get that $\{y \in X:(\exists f \in G): y \circ \pi \supset f\} \in F$. Let $R=\{y \in$ $X:(\exists f \in Q): y \circ \pi \supset f\} \cap \bigcap\{\{y \in X:(\exists f \in G) y \circ \pi \supset f\}: G \in \Gamma\} . R$ is the intersection of fewer than $\mu$ sets in $F$ and so $R \in F$. We must show that $\{y \in X:(\exists x \in A):(\forall s \in \phi(X)) y(\pi(s))=1 \Leftrightarrow x \in s\} \in F$. It suffices to show that this set contains $R$. To see this, suppose $y \in R$. There must exist $f \in Q$ such that $y \circ \pi \supset f$. There must also be a node $(B, f) \in E$. We now examine the reason that $(B, f)$ was decided to have no successors. If $\varnothing \in G$, at that time, then there is $x \in B$ such that $\Psi(x)=f$ and so $y \circ \pi \supset \psi(x)$. If $|G|=\mu$, at that time, then $G \in \Gamma$ and so, if $\exists g \in G$ such that $y \circ \pi \supset g$, then there is $x \in B$ such that $y \circ \pi \supset \Psi(x)-f$. Since $y \circ \pi \supset f$, we have $y \circ \pi \supset \Psi(x)$. In each case, there is $x \in B$ such that $y \circ \pi \supset \Psi(x)$, that is, such that $(\forall s \in \phi(X)) y(\pi(S))=1 \Leftrightarrow x \in S$.

Next, we show condition 4 of Lemma 2. Let $\left\{x_{i}^{\alpha}: \alpha \in \mu, i \in 2\right\}$ be distinct elements of $X$ where $\mu \leq \kappa$. For each $\alpha \in \mu$, let $f_{\alpha}=\left\{\left(\left\{x_{0}^{\alpha}\right\}, 1\right),\left(\left\{x_{1}^{\alpha}\right\}, 1\right)\right\}$. Thus $\left\{f_{\alpha}: \alpha \in \mu\right\} \in\left[F n\left(2^{X}, 2\right)\right]^{\mu}$. By hypothesis 6 of the lemma, $Y=\{y \in$ $\left.X:(\exists \alpha \in \mu): y \circ \pi \supset f_{\alpha}\right\} \in F . F$ is uniform and so we can use $Y$ instead of $X$ (and $\pi \uparrow 2^{Y}$ instead of $\pi$ ) in Lemma 2. Whenever $x_{0}, x_{1} \in Y$, there is $\alpha \in \mu$ such that $x_{1} \circ \pi \supset f_{\alpha}$. Therefore $(\forall i \in 2) x_{1}\left(\pi\left(\left\{x_{i}^{\alpha}\right\}\right)\right)=1$. There is $i \in 2$ such that $x_{0} \neq x_{i}^{\alpha}$ and thus exactly one of $x_{1}\left(\pi\left(\left\{x_{i}^{\alpha}\right\}\right)\right)=1$ and $x_{0} \in\left\{x_{i}^{\alpha}\right\}$ is true.

We shall now produce $F$ and $X$. In reading the next lemma, the reader should interpret $F P$ to be designed to take care of the $\mu$-sized families with disjoint domains and interpret $B$ to be designed to take care of the families of size less than $\mu$ (although it does this only under the assumption that $F$ is an ultrafilter). In this lemma we only assume that $F$ is a filter. $A$ will eventually be the ultrafilter although it is used to define the filter whence the ultrafilter arises.

We really need $X$ to be "random". The Hewitt-Marczewski-Pondiczery theorem does this for us under $\sup \left\{\kappa^{\nu}: \nu<\kappa\right\}=\kappa$. This method of getting a "random" set is used in [7] as well.

Lemma 4. Let $\kappa$ be a cardinal such that $\sup \left\{\kappa^{\nu}: \nu<\kappa\right\}=\kappa$ and let $\lambda=2^{\kappa}$. Let $A \subset \lambda$ be such that $|A|=|\lambda-A|$. There is $X \in\left[{ }^{\lambda} 2\right]^{\kappa}$ such that, letting $F P=\left\{Q \in[F n(\lambda, 2)]^{\kappa}: \operatorname{dom}(Q)\right.$ is a pairwise disjoint family $\}$ and letting $B=\{\{g \in X:(\exists f \in Q): g \supset f\}: Q \in F P\} \cup\{\{g \in X: g(a)=1\}: a \in$ $A\} \cup\{\{g \in X: g(a)=0\}: a \in \lambda-A\}, D \in[B]^{<\kappa}$ implies $\cap D \neq \varnothing$.

Proof. Using the Hewitt-Marczewski-Pondiczery theorem in a more general form (see 2.3.15 of [2]) let $X \in\left[{ }^{\lambda} 2\right]^{\kappa}$ be such that, whenever $f$ is a partial function from $\lambda$ into 2 of cardinality less than $\kappa$, there is $x \in X$ such that $x \supset f$. We must show that $D \in[B]^{<\kappa}$ implies $\bigcap D \neq \varnothing$. Let $D=\{\{g \in$ $\left.\left.X:\left(\exists f \in Q_{i}\right): g \supset f\right\}: i \in I\right\} \cup\left\{\{g \in X: g(a)=1\}: a \in A_{0}\right\} \cup\{\{g \in X: g(a)=$ $\left.0\}: a \in B_{0}\right\}$ where $\left\{Q_{i}: i \in I\right\} \subset F P$ and $|I|<\kappa$ and $A_{0} \in[A]^{<\kappa}$ and $B_{0} \in[\lambda-A]^{<\kappa}$. By induction, we can find, for each $i \in I, f_{i} \in Q_{i}$ such that if $i, j$ are distinct then $\operatorname{dom}\left(f_{i}\right) \cap \operatorname{dom}\left(f_{j}\right)=\varnothing$ and for each $i \in I$, 
$\operatorname{dom}\left(f_{i}\right) \cap\left(A_{0} \cup B_{0}\right)=\varnothing$. Now let a partial function $f^{*}: \lambda \rightarrow 2$ be defined by $\operatorname{dom}\left(f^{*}\right)=A_{0} \cup B_{0} \cup \bigcup\left\{\operatorname{dom}\left(f_{i}\right): i \in I\right\}$ and $f^{*}\left|A_{0} \equiv 1 ; f^{*}\right| B_{0} \equiv 0 ; f^{*} \uparrow$ $\operatorname{dom}\left(f_{i}\right)=f_{i}$. Now $\left|\operatorname{dom}\left(f^{*}\right)\right|<\kappa$ implies that there is $x \in X$ such that $x \supset f^{*}$. Thus $x \in \cap D$ and the proof is complete.

We can now use the ultrafilter which the compact cardinal provides to simplify the difficult requirement of Lemma 3 and prove the main result.

Theorem 1. If there is a strongly compact cardinal $\kappa$, then there is a $\sigma$-discrete hereditarily normal Dowker space.

Proof. That $\kappa$ is a strongly compact cardinal says that any $\kappa$-complete filter can be extended to a $\kappa$-complete ultrafilter. The inaccessibility of $\kappa$ implies that $\sup \left\{\kappa^{\nu}: \nu<\kappa\right\}=\kappa$ and so we can let $\lambda, A, X$ and $B$ be as in Lemma 4. We use strong compactness to get a $\kappa$-complete ultrafilter $F$ which includes the $\kappa$-complete filter base $B$. We shall fulfill the hypothesis of Lemma 3 with $\mu=\kappa$. Let $\pi: 2^{X} \rightarrow \lambda$ be a bijection such that $\pi(F)=A$. Let $Q \subset F n\left(2^{X}, 2\right)$. If $|Q|<\mu$ and $\bigcup\left\{\cap f^{-1}(1)-\bigcup f^{-1}(0): f \in Q\right\} \in F$ then, by $\mu$-completeness, there is $f \in Q$ such that $\bigcap f^{-1}(1)-\bigcup f^{-1}(0) \in F$. Now $f(a)=1$ implies $a \in F$ which implies $\pi(a) \in A$ which implies $\{g \in X: g(\pi(a))=1\} \in B \subset$ $F$. Similarly, $f(a)=0$ implies $\{g \in X: g(\pi(a))=0\} \in F$. Therefore $\{g \in X: g \circ \pi \supset f\} \in F$ which is more than we need. If $|Q|=\mu$ and the elements of $Q$ have pairwise disjoint domains, then since $\pi$ is one-to-one $\left\{f \circ \pi^{-1}: f \in Q\right\} \in F P$ and $\left\{g \in X:(\exists f \in Q): g \supset f \circ \pi^{-1}\right\}=\{g \in X:(\exists f \in$ $Q): g \circ \pi \supset f\} \in F$ as required.

\section{ACKNOWLEDGMENT}

The author would like to thank Mary Ellen Rudin for discussing this paper at length. The proof of Lemma 1 is due to her.

\section{REFERENCES}

1. R. H. Bing, Metrization of topological spaces, Canad. J. Math. 3 (1951), 175-186.

2. R. Engelking, General topology, Polish Scientific Publishers, 1977.

3. A. Kanamori and M. Magidor, The evolution of large cardinal axioms in set theory, Springer Lecture Notes, vol. 669, 1978, pp. 99-276.

4. Kenneth Kunen, Set theory, North-Holland, Amsterdam, 1983.

5. Mary Ellen Rudin, Dowker spaces (K. Kunen and J. Vaughan, eds.), Handbook of Set-Theoretic Topology, North-Holland, Amsterdam, 1984, Chapter 17, pp. 761-781.

6. Lectures in set-theoretic topology, Regional Conference Series in Mathematics, vol. 23, Amer. Math. Soc., Providence, RI, 1975.

7. Stephen Watson, Separation and coding, Trans. Amer. Math. Soc. (to appear).

Department of Mathematics, York University, North York, Ontario M3J 1P3 CANADA 